\title{
Ein Verfahren zum oxydativen Nachweis von organischer Substanz im Seewasser
}

\author{
Von Max Gillbricht \\ Aus der Biologischen Anstalt Helgoland, List auf Sylt, \\ in der Bundesforschungsanstalt für Fischerei \\ (Mit 7 Abbildungen)
}

Im Seewasser sind in recht beträchtlicher Menge organische Stoffe vorhanden (ca. $2 \mathrm{mg} \mathrm{C/1}$ ). Nach unserer heutigen Auffassung (KAY, 1954, mit weiteren Literaturangaben) sind diese zur Hauptsache (mindestens zu $90 \%$ ) gelöst. Der Rest verteilt sich dann in stark wechselndem Verhältnis auf Detritus und lebendes Plankton, wobei das Plankton wenigstens in den Küstengewässern zumeist hinter dem Detritus zurückstehen dürfte (Grllbricht, 1952). Wir bestimmen folglich mit den organischen Substanzen im Seewasser zur Hauptsache den gelösten Anteil; bei nicht zu empfindlichen Methoden liefern Differenzmessungen zwischen filtriertem und unfiltriertem Seewasser erst bei größeren Probenzahlen auswertbare Unterschiede.

Diese organische Substanz wird zweckmäßig durch ihren Kohlenstoffgehalt charakterisiert und entsprechend nachgewiesen. Leider stören hierbei die Salze des Seewassers sehr (KAY, 1954 a). Der Analysengang wird entsprechend umständlich, so daß sich größere Meßreihen von selber verbieten.

Einen Einblick in die Mengenverteilung der organischen Substanz kann man aber nur durch Aufarbeitung zahlreicher Proben gewinnen. Es erschien daher angebracht, nach einer einfacheren Nachweismethode Ausschau zu halten. Als für unseren $Z$ weck geeignet erwies sich nach systematischen Untersuchungen eine Modifikation des von RUPPIN (1904) vorgeschlagenen oxydativen Verfahrens. Diese Methode gibt dann natürlich nur Oxydationswerte, und eine Angabe in $\mathrm{mg} \mathrm{C} / 1$ ist nicht möglich. Aus Gründen der Anschaulichkeit dürfte es jedoch vertretbar sein, von $\mathrm{C}$-Äquivalenten zu sprechen, $d . h$. den Oxydationswert durch die äquivalente C-Menge unter der Annahme von Kohlenhydraten auszudrücken. Eine weitere Unsicherheit liegt in der unvollständigen Oxydation, mit der man wohl bei jeder nassen Verbrennung rechnen muß (Sverdrup, Johnson und Fleming, 1946).

\section{Der Reaktionsverlauf}

RUPPin (1904) kocht die Seewasserprobe nach Zugabe von $\mathrm{NaOH}$ und $\mathrm{KMnO}_{4}$ auf. Hierbei geht bei Anwesenheit von oxydierbaren Substanzen das Permanganat über das Manganat zum Braunstein über: 


$$
\begin{aligned}
& {\left[\mathrm{MnO}_{4}\right]^{\prime}+\mathrm{e}=\left[\mathrm{MnO}_{4}\right]^{\prime \prime}} \\
& {\left[\mathrm{MnO}_{4}\right] "+4 \mathrm{H}^{\prime \prime}+2 \mathrm{e}=\mathrm{MnO}_{2}+2 \mathrm{H}_{2} \mathrm{O}}
\end{aligned}
$$

Dieser Ablauf ist jedoch nur in stark alkalischer Lösung zu beobachten. Wird wenig Natronlauge zugesetzt, dann ist die H-Ionen-Konzentration noch hinreichend, gemäß der Bruttoformel

$$
\left[\mathrm{MnO}_{4}\right]^{\prime}+4 \mathrm{H}^{\circ}+3 \mathrm{e}=\mathrm{MnO}_{2}+2 \mathrm{H}_{2} \mathrm{O}
$$

die direkte Bildung von Braunstein neben Permanganat zu bewirken. In saurer Lösung ist bekanntlich auch der Braunstein nicht mehr beständig, und wir erhalten:

$\left[\mathrm{MnO}_{4}\right]^{\prime}+8 \mathrm{H}^{\cdot}+5 \mathrm{e}=\mathrm{Mn}^{*}+4 \mathrm{H}_{2} \mathrm{O}$.

Diese letzte Reaktionsform ist jedoch im Seewasser nicht anwendbar, da unter dieser Bedingung das in großer Menge vorhandene Chlorid zu Chlor oxydiert wird, das zum großen Teil entweicht, so daß der Oxydationswert verlorengeht. Auch beim Arbeiten in einem abgeschlossenen System entstehen gemäß

$$
2 \mathrm{Cl}_{2}+2 \mathrm{H}_{2} \mathrm{O} \gtreqless 4 \mathrm{HCl}+\mathrm{O}_{2}
$$

unkontrollierbare Verluste. Im alkalischen Milieu werden die sich in geringer Menge bildenden Oxydationsprodukte des Chlorids in heißer Lösung sofort als Chlorat gebunden.

Nun werden jedoch durch die alkalische Permanganatlösung einige Stoffe (Kohlenhydrate, Cellulose) nur bis zur Oxalsäure abgebaut, so daß man die Probe zuletzt für kurze Zeit vorsichtig nach Abkühlung ansäuern muß, wodurch zunächst alle Reduktionsprodukte des Permanganats in Permanganatund Mangan (II)-Ionen zerfallen:

$$
\begin{aligned}
& 5\left[\mathrm{MnO}_{4}\right]^{\prime \prime}+8 \mathrm{H}^{\circ}=4\left[\mathrm{MnO}_{4}\right]^{\prime}+\mathrm{Mn}^{*}+4 \mathrm{H}_{2} \mathrm{O} \\
& 5 \mathrm{MnO}_{2}+4 \mathrm{H}^{*}=2\left[\mathrm{MnO}_{4}\right]^{\prime}+3 \mathrm{Mn}:+2 \mathrm{H}_{2} \mathrm{O} .
\end{aligned}
$$

Sind die geringen Mengen Oxalsäure nach kurzer Zeit oxydiert, dann wird Jodidlösung zugesetzt. So wird der Oxydationswert des Permanganats gemäß Gleichung (4) und der des Chlorats bzw. des zum Teil schon entstandenen Chlors zur Oxydation von Jodid zu Jod benutzt.

Die Menge des so erhaltenen freien Jods läßt sich wie üblich mit Thiosulfat unter Zusatz eines Indikators titrimetrisch bestimmen.

\section{Die Ausführung der Messungen}

In $50 \mathrm{ml}$ Jenaer Glasstopfenflaschen wird zunächst (u. U. schon vor Antritt der Reise) je $1 \mathrm{ml}$ verdünnte $\mathrm{H}_{2} \mathrm{SO}_{4}$ pipettiert ( $250 \mathrm{ml}$ aqua dest. $+30 \mathrm{ml}$ $\mathrm{H}_{2} \mathrm{SO}_{4}$ ), wodurch eine Zersetzung der organischen Substanz verhindert wird. Eine sofortige Zugabe von Natronlauge ist nicht ratsam, wenn die Probe einige Zeit stehen soll. Der Hydroxydniederschlag altert dann, was die weitere Verarbeitung erschwert. In die mit Säure versetzten Flaschen werden jeweils $15 \mathrm{ml}$ Seewasser sofort nach der Probennahme gefüllt. Zum Abmessen wird zweckmäßig eine Knudsenpipette für die Chlortitration benutzt. Nach kurzem Umschwenken ist die Probe lagerfähig.

Später erfolgt die Oxydation unmittelbar in der Flasche selber, wodurch Verunreinigungen und Substanzverluste weitgehend vermieden werden, durch Zugabe von $1 \mathrm{~g} \mathrm{NaOH}$ in Plätzchen und nach deren Auflösung von $5 \mathrm{ml}$ Permanganatlösung (zweckmäßig mit einer Knudsenpipette), deren Konzentration je nach der zu erwartenden Kohlenstoffmenge wie folgt zu wählen ist: 


\begin{tabular}{cccc} 
bis mg C-Äquivalent/1 & $\begin{array}{c}\mathrm{KMnO}_{4} \\
{[\mathrm{val} / 1]}\end{array}$ & $\mathrm{ml}$ & $\mathrm{KJ}$ \\
\hline 3 & $1 / 100$ & 1 & 3 \\
7 & $1 / 50$ & 1 & 5 \\
17 & $1 / 20$ & 2 & 5
\end{tabular}

Die so vorbehandelte Lösung wird jetzt nach Umschwenken für eine Stunde in siedendes Wasser gestellt. Hierzu wird der Glasstopfen zunächst abgenommen, kann aber nach wenigen Sekunden wieder aufgesetzt werden. Mit Rücksicht auf den Hydroxydniederschlag empfiehlt es sich, die Probe nach einiger Zeit (ca. 15 Min.) nochmals kurz umzuschwenken.

Wenn die Probe sehr bald aufgearbeitet werden soll, kann man auf die Zugabe der verdünnten Schwefelsäure verzichten und der Wasserprobe sofort $0,8 \mathrm{~g} \mathrm{NaOH}$ zusetzen. Die Verwendung flüssiger Natronlauge ist unangenehmer und erhöht die Genauigkeit der Messung nicht.

Ist die Kochzeit beendet, so kühlt man die Probe (evtl. in kaltem Wasser) auf Zimmertemperatur ab; dann wird sie nach Umschwenken mit $30 \mathrm{ml}$ einer wie folgt bereiteten, stark verdünnten Schwefelsäure versetzt:

Zunächst werden 3 Vol. aqua dest. und 1 Vol. $\mathrm{H}_{2} \mathrm{SO}_{4}$ gemischt. Von dieser $\mathrm{H}_{2} \mathrm{SO}_{4}(1: 3)$ werden jeweils $30 \mathrm{ml} \mathrm{zu} 250 \mathrm{ml}$ aqua dest. zugesetzt, um so die Gebrauchslösung zu erhalten. Bei der zweiten Verdünnung ist keine Erwärmung mehr zu beobachten, so daß sie auch unmittelbar vor Benutzung vorgenommen werden kann.

Eine halbe bis eine Minute später wird die KJ-Lösung zugegeben, die Flasche verschlossen und kurz umgeschwenkt.

Da Jodid leicht zu Jod oxydiert wird, ist die KJ-Lösung mit großer Sorgfalt zu bereiten und in braunen Flaschen (bei Nichtgebrauch im Dunkeln) aufzuheben. Eine übertriebene Vorratswirtschaft ist zu vermeiden. Die Pipette muß nach jeder Probenserie mit destilliertem Wasser ausgespült werden, da sich sonst die Jodidreste an ihr zersetzen.

Frühestens zehn Minuten nach Zugabe des Jodids, während welcher Zeit die Flasche im Dunkeln stehen muß, kann die Titration mit Thiosulfatlösung $(1 / 100 \mathrm{val} / \mathrm{l})$ unter Zusatz von $1 \mathrm{ml}$ Stärkelösung $(1 \%)$ erfolgen. Hierfür wird zweckmäßig eine $25 \mathrm{ml}$-Bürette benutzt (Teilung $1 / 20 \mathrm{ml}$ ).

OHLE (1953) empfiehlt außerdem als Indikator Polyviol; zur Stabilisierung des Titers der Thiosulfatlösung schlägt er die Zugabe von Natriumhydroxyd (HAHN und Windisch, 1922) und von Isobutylalkohol (WINKLER, 1931) vor.

Jetzt wird der Verlust an Oxydationswert gegenüber einer gleich behandelten Kontrolle mit aqua dest. ermittelt. Schließlich wird der Titer der Thiosulfatlösung mittels $5 \mathrm{ml}$ Jodlösung $(1 / 20 \mathrm{val} / \mathrm{l})$ bestimmt (KaLLE, 1939).

$$
\begin{array}{ll}
\text { Berechnung: } & \mathrm{T}=\text { Titrationswert der Jodlösung } \\
\mathrm{K}=\text { Titrationswert der Kontrolle } \\
\mathrm{P}=\text { Titrationswert der Probe } \\
\\
\mathrm{mg} \mathrm{C-Aquivalent/1}=50,0 \cdot \frac{(\mathrm{K}-\mathrm{P})}{\mathrm{T}} \\
& \mathrm{mg} \mathrm{KMnO}_{4} / 1=527 \cdot \frac{(\mathrm{K}-\mathrm{P})}{\mathrm{T}}
\end{array}
$$

Die Angabe als Permanganatverbrauch ist bei der angewandten Methodik als korrekter zu erachten, weshalb die entsprechende Formel hier angeführt ist.

Es ist darauf zu sehen, daß $\frac{(K-P)}{K} \leqq 1 / 3$ ist (s. S. 79); wird dieser Wert überschritten, muß die Probe verworfen werden. 
Helgol. Wiss. Meeresunters. 6, l : Gillbricht
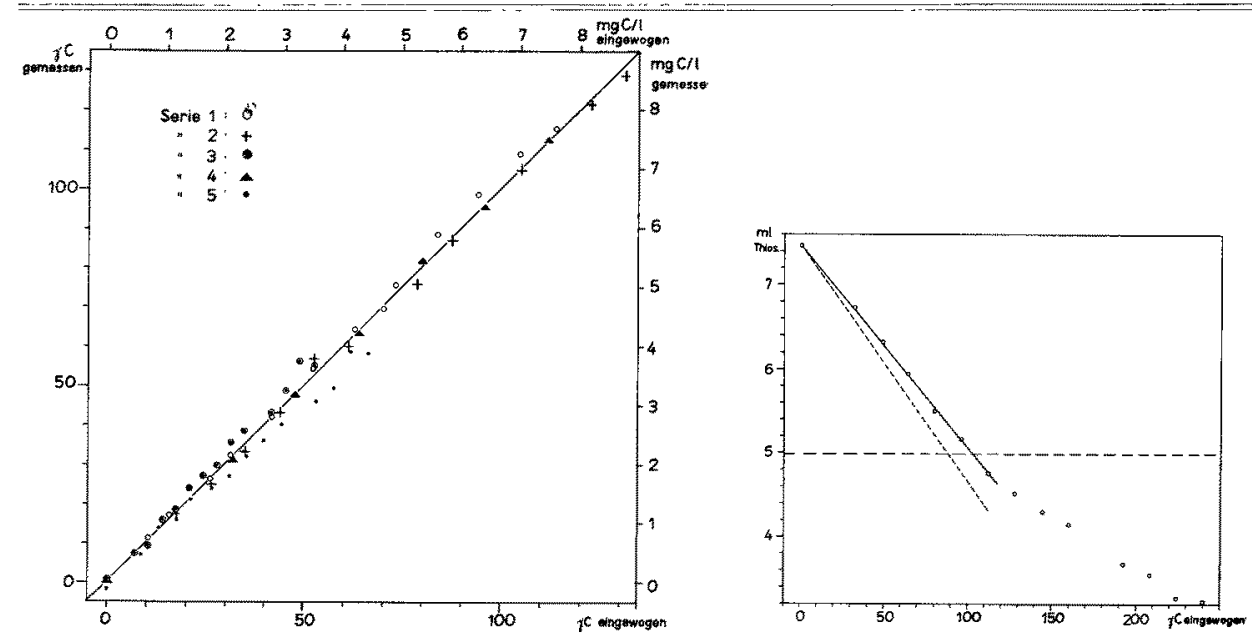

Abb. 1. Zusammenhang zwischen den Einwaagen und den umgerechneten Meßwerten der Eichungen.

Abb. 2. Zusammenhang zwischen Thiosulfatverbrauch und Einwaage bei der vierten Eichserie. Die ausgezogene Gerade ist durch die Meßpunkte festgelegt, während dic unterbrochene den theoretisch zu fordernden Verlauf anzeigt. Bis zur Waagrechten ist gerade ein Drittel des Oxydationswertes des Permanganats verbraucht worden.

Abb. 3. Zehrungsversuch. mg G-Aquivalent/l: $\mathrm{O}_{2} ;---$. Der Sauerstoff ist wegen des besseren Vergleichs ebenfalls als die ihm äquivalente Kohlenstoffmenge angegeben. Dreifach übergreifende Mittel. (Bei dieser Mittelung mußte ein Kohlenstoffwert als offensichtliche Fehlmessung verworfen werden.)

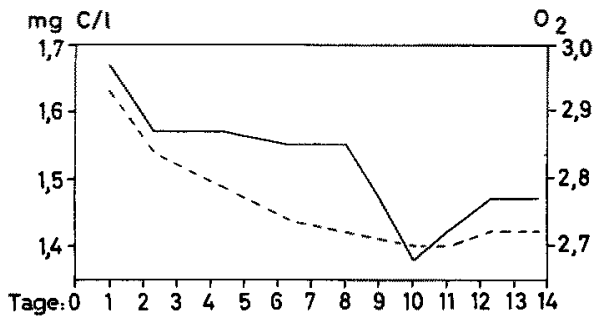

Abb. 4. Lineare Abhängigkeit des Kohlenstoffäquivalents vom Saverstoffgehalt beim Zehrungsversuch. Die bei quantitativem Nachweis der veratmeten Substanz resultierende Gerade ist unterbrochen eingetragen.

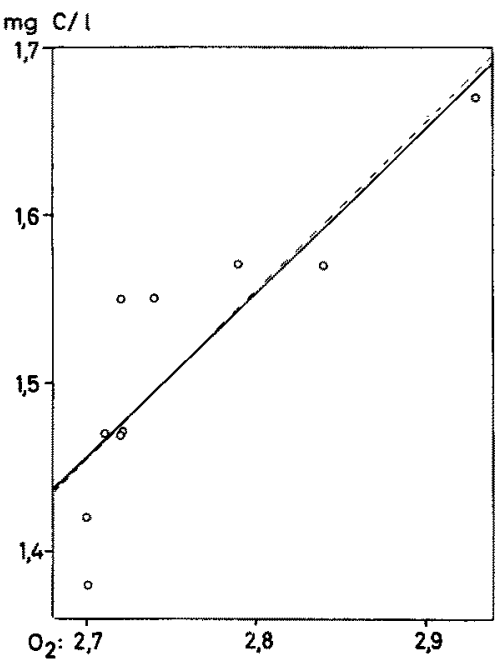


An anorganischen Stoffen kann im Seewasser praktisch nur $\mathrm{H}_{2} \mathrm{~S}$ stören. Die Probe muß dann zunächst angesäuert offen bis zur Vertreibung des Schwefelwasserstoffs stehen, was durch Erwärmen im Wasserbad beschleunigt werden kann.

\section{Bemerkungen zur Methodik}

Es erschien zunächst fragwürdig, ob eine Kontrollmessung mit aqua dest. als Bezugswert angesehen werden könnte; jedoch ließ sich zeigen, daß das von uns benutzte destillierte Wasser keine nachweisbaren Mengen an durch Permanganat oxydierbarer Substanz enthielt. Diese Voraussetzung dürfte bei Verwendung von Wasser aus guten modernen Destilliergeräten wohl meist gegeben sein. (Wir benutzten den Wasserdestillationsapparat aus Hanauer Quarzglas.)

Der Nachweis wurde wie folgt geführt: Aqua dest. wurde mit etwas $\mathrm{H}_{2} \mathrm{SO}_{4}$ und einer Spur Permanganat versetzt und aufgekocht, das überschüssige Permanganat wurde durch etwas Salzsäure zerstört, Chlor und Salzsäure wurden verkocht. Entsprechend der zugesetzten Menge Schwefelsäure wurde mehr $\mathrm{NaOH}$ zugegeben. Für zwei solcher Wasserproben ergab sich:

\begin{tabular}{ccc}
$\mathrm{g} \mathrm{NaOH} /$ Probe & ml Thiosulfatlösung & Mittel \\
\hline 1,0 & 4,38 & $4,363 \pm 0,012 \mathrm{ml}$ \\
1,0 & 4,34 & \\
1,0 & 4,37 & $4,053 \pm 0,018 \mathrm{ml}$ \\
\hline 1,4 & 4,06 & \\
1,4 & 4,02 & \\
1,4 & 4,08 &
\end{tabular}

Aus diesen Zahlen ermittelt sich durch lineare Extrapolation für die NaOH-Menge $0,8 \mathrm{~g}: 4,518 \pm 0,027 \mathrm{ml}$. Eine gleichzeitige Doppelbestimmung mit unserem unbehandelten destillierten Wasser ergab: $4,555 \pm 0,005 \mathrm{ml}$. Das Wasser muß folglich als einwandfrei angesehen werden, während das $\mathrm{NaOH}$ eine erhebliche reduzierende Wirkung hat.

Ferner ließ sich durch Vergleichsmessungen zeigen, daß weder $\mathrm{NaCl}$ noch $\mathrm{NaCl}+\mathrm{MgSO}_{4}$ in aqua dest. den Verbrauch an Thiosulfatlösung verändern. Die Salze des Seewassers dürtten also ohne Einfluß sein.

Die Vollständigkeit der Oxydation wurde für Kohlenhydrate (Rohrzucker und Stärke) in fünf Meßreihen in aqua dest. und Seewasser untersucht. Für den Oxydationsgrad ergab sich: $85,7 \pm 2,0 \%$. Nach diesem Befund wäre es angebracht, die gemessenen Werte mit dem empirischen Faktor $F=1,17 \mathrm{zu}$ multiplizieren. Nach dieser Umrechnung ergibt sich für alle Eichpunkte innerhalb des Proportionalitätsbereichs der in Abb. 1 gezeigte Zusammenhang. Nun muß man außerdem wissen, welcher Uberschuß an Permanganat zur Erreichung dieses Oxydationsgrades notwendig ist. Nach den vorliegenden Messungen sollte bei Zufügung einer Permanganatmenge, deren (saurer) Oxydationswert bis zu einem Drittel ausgenutzt wird, eine hinreichende Sicherheit hierfür gegeben sein (Abb. 2).

Um den Oxydationsgrad bei den im freien Wasser vorkommenden Stoffen zu untersuchen, wurde ein Zehrungsversuch angesetzt (Abb. 3). Das Seewasser $(\mathrm{S}=29,8 \%$ ) wurde am 2. 6. 1956 in der Nähe von List geschöpft, in eine 10-I-Flasche gefüllt und zum Zwecke des Luftabschlusses mit Paraffinöl überschichtet. Dann wurde die Flasche im Keller in völliger Dunkelheit bei einer Temperatur von 14 bis $15^{\circ}$ aufgehoben. Vom 4.6. ab wurden während 
eines Zeitraums von 15 Tagen Wasserproben für die $\mathrm{O}_{2}$ - und $\mathrm{C}$-Bestimmung abgehebert. Nun ist nicht zu erwarten, daß sich besonders saubere Kurven ergeben, da hinsichtlich der Zehrungsvorgänge mit Schichtungen gerechnet werden muß (Bakterienwachstum an den Wandungen). So zeigt denn trotz der Mittelbildung vor allem die Kohlenstoffkurve einen recht unausgeglichenen Verlauf, der aber sicherlich nur rein zufällig ist. Insgesamt decken sich jedoch die beiden Kurven recht gut. Die in Abb. 4 dargestellte lineare Abhängigkeit des Kohlenstoffgehaltes vom Sauerstoff spricht innerhalb der Fehlergrenzen für einen vollständigen Nachweis der veratmeten organischen Stoffe. Die Anwendung des oben erhaltenen Korrektionsfaktors auf Messungen im natürlichen Seewasser erscheint daher nicht angebracht.

Der mittlere Fehler der Methode hat sich in zahlreichen Mehrfachbestimmungen zu $\pm 1 \gamma \mathrm{C}$-Äquivalent/Probe bzw. $\pm 0,07 \mathrm{mg} \mathrm{C}$ - đ̈quivalent/l ergeben. Dieser Wert gilt bei Verwendung einer Permanganatlösung mit $1 / 100$ oder $1 / 50$ $\mathrm{val} / \mathrm{l}$, bei einer solchen mit $1 / 20 \mathrm{val} / 1$ ist mit einem um $10 \%$ höheren Betrag zu rechnen.

Dieser Fehler ist für den Vergleich innerhalb einer Meßreihe ermittelt worden. Da die Streuung bei Kontrolle und Messung gleich ist, ergibt sich für den Absolutwert, wenn jeweils zwei Kontrollwerte vorliegen:

$$
\mathrm{f}= \pm\left(0,07+\frac{0.07}{\sqrt{2}}\right) \mathrm{mg} \mathrm{C} \text {-Äquivalent } / 1= \pm 0,12 \mathrm{mg} \mathrm{C} \text {-Äquivalent } / 1
$$

Mögliche Ungenauigkeiten bei der Einstellung der Thiosulfatlösung gegen eine bekannte Jodlösung fallen demgegenüber nicht ins Gewicht, so daß die Ergebnisse auf $0,1 \mathrm{mg} \mathrm{C}$-Äquivalent/1 genau gebracht werden können. Es ist sehr fraglich, ob selbst eine wesentliche Erhöhung der Wassermenge die Angabe der zweiten Dezimale rechtfertigen würde.

\section{Anwendungsbeispiel}

Auf einer Fahrt von FFS „Anton Dohrn“ in die südwestliche Nordsee im Juni/Juli 1956 wurden auf einem Schnitt von Texel über Outer Silver Pit und Doggerbank Proben zur Bestimmung der gesamten oxydierbaren Substanz genommen (Abb.5), die durch Oberflächenwerte von Chlorophyll und Eiweiß ergänzt wurden $\left.{ }^{1}\right)$.

Die Mengen an Chlorophyll und Eiweiß nehmen danach von der Küste fort $a b(\mathrm{Abb} .6 \mathrm{a})$, das Isoplethendiagramm des Kohlenstoffäquivalents zeigt dagegen eine ganz andere Verteilung (Abb. 6b). Dichter unter Land finden sich die geringsten Werte, weiter draußen eine erhebliche Zunahme, bei Outer Silver Pit ein Minimum und jenseits der Doggerbank eine sehr große Menge. Leider war mit so hohen Werten nicht gerechnet und eine schwache Permanganatlösung benutzt worden, so daß nur bis $4 \mathrm{mg}$ G-Äquivalent/ 1 Isolinien eingezeichnet werden konnten. Auffallend ist, daß lediglich in der Nähe der Küste keine vertikale Schichtung zu beobachten ist, von Station 942 ab ist das

1) Die Untersuchung wurde mit Mitteln der Deutschen Wissenschaftlichen Kommission für Meeresforschung durchgeführt, für die Überlassung des zur Chlorophyll- und Eiweißbestimmung notwendigen ELKO II (Zeiss) danke ich der Deutschen Forschungsgemeinschaft. Chlorophyll wurde nach KREY (1939) und EiweiB nach KREX (1952) bestimmt. Herr Prof. Dr. KREY überließ mir freundlichst seine neuesten Eidhwerte für die Chlorophyll- und Eiweißmessungen mit dem Elko. Die Daten für Temperatur und Salzgehalt verdanke ich dem Deutschen Hydrographischen Institut. 
Helgol. Wiss. Meeresunters. 6, 1 : Gillbricht

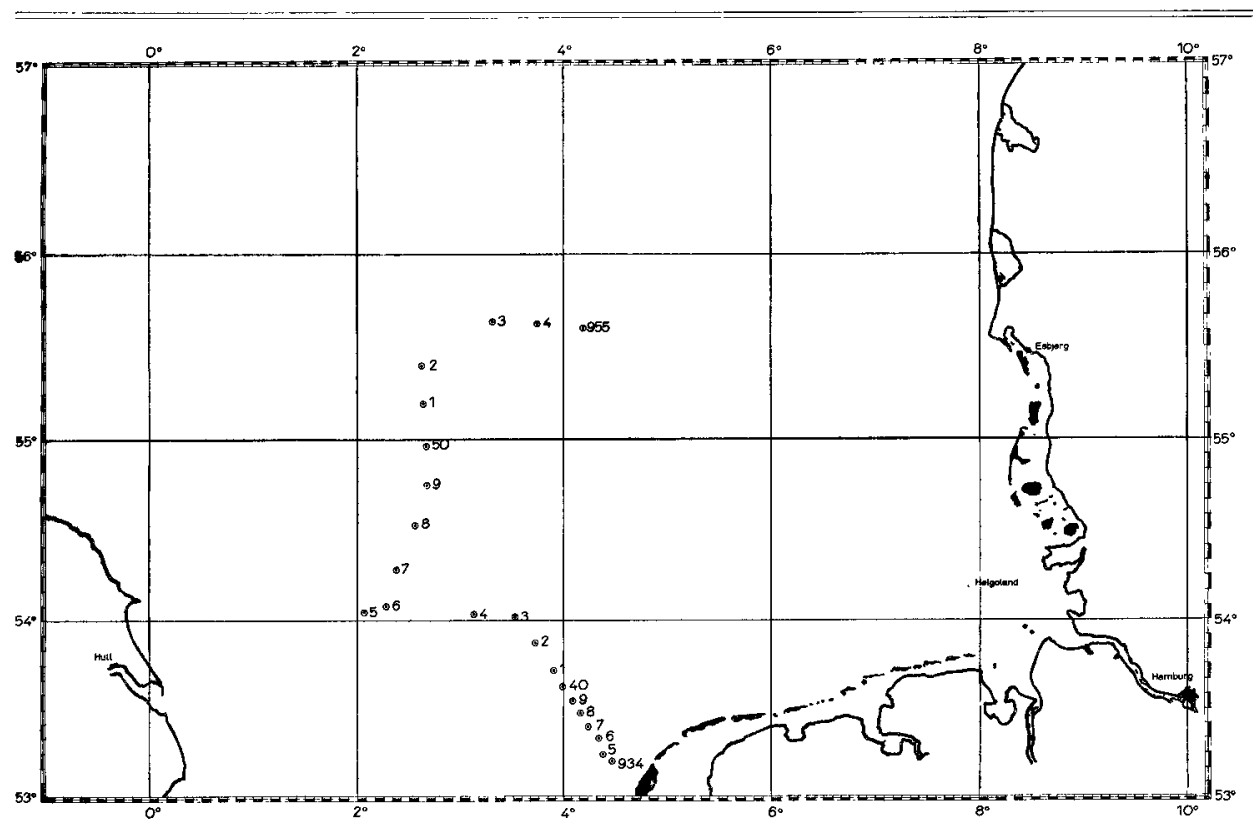

Abb. 5. Ubersichtskarte über die Lage der Stationen 934 bis 955 von FFS „Anton Dohrn“ 29. 6. bis 1. 7. 1956.
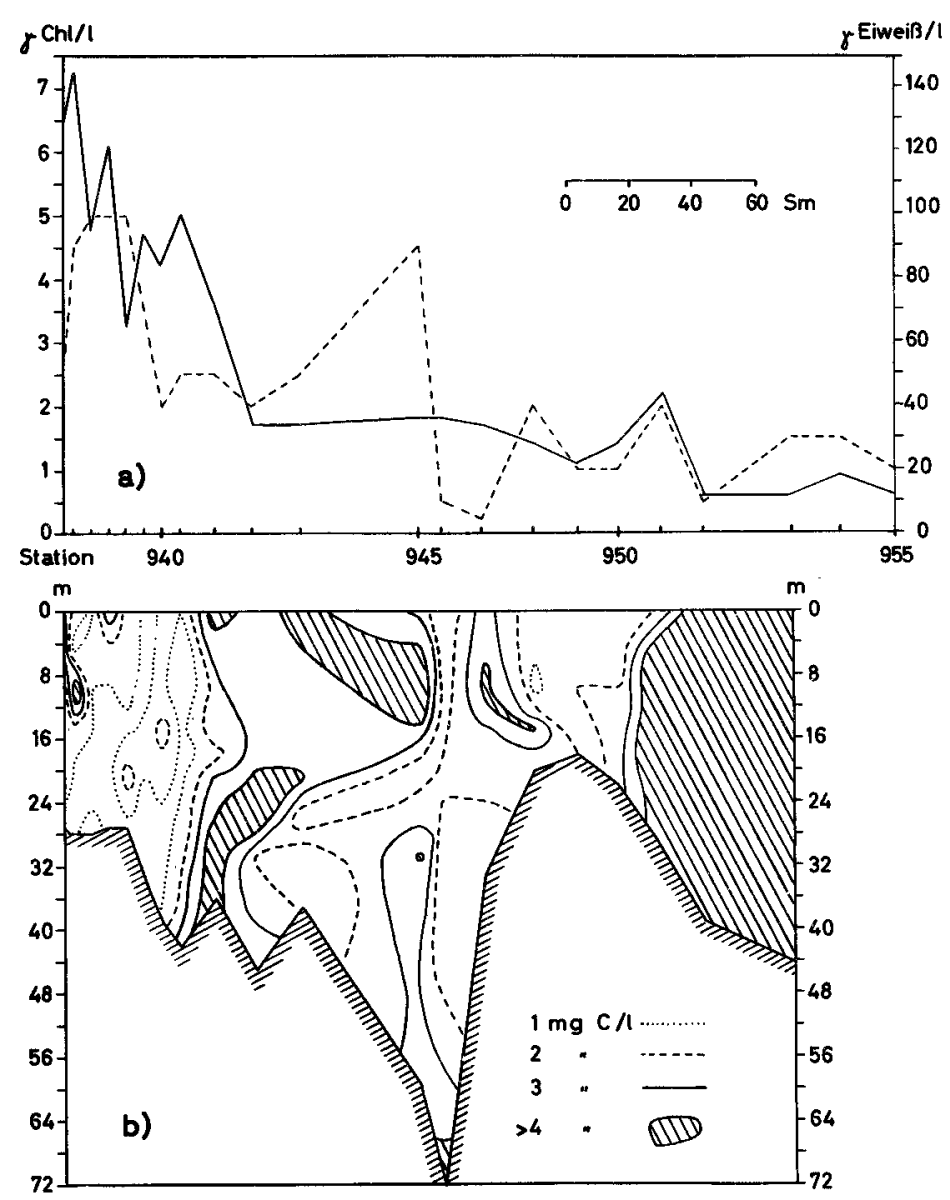

Abb. 6. Stationen 934 bis 955 von FFS „Anton Dohrn“

a) Oberflächenwerte von Chlorophyll (_- ) und Eiweiß (- - -),

b) Isoplethendiagramm des Kohlenstoffäquivalents. 
Wasser aber hinsichtlich des Gehalts an organischer Substanz sehr heterogen. Dies läßt auf das Vorhandensein biologisch verschiedener Wasserkörper schließen, deren gegenseitige Vermischung demnach nicht sehr groß sein kann, die sich jedoch hydrographisch (Temperatur und Salzgehalt) nicht nachweisen lassen.

In anderen Jahren wurden in diesem Gebiet durchaus thermisch und halin unterscheidbare Wasserkörper gefunden (DiETRICH, 1954), nur waren in diesem Jahr die Unterschiede offenbar zufällig zu gering.

Recht aufschlußreich ist nunmehr ein C/S-Diagramm (Abb. 7), hier zeichnen sich deutlich drei Wasserarten ab. Das salzarme Wasser an der Küste (I) enthält wenig Kohlenstoff, dessen Menge mit zunehmendem Salzgehalt etwas abnimmt. Ein mittlerer Wasserkörper (II) zeigt höhere Salz- und Kohlenstoffwerte, die Menge der organischen Substanz nimmt mit zunehmendem Salzgehalt stark ab. Dann ist noch ein salzreiches Wasser (III) angeschnitten worden, dessen gemessene C-Werte aus dem oben (S. 81) angeführten Grunde fraglos viel zu niedrig sind, das sich aber auf jeden Fall biologisch deutlich von den beiden anderen Wasserarten abhebt.

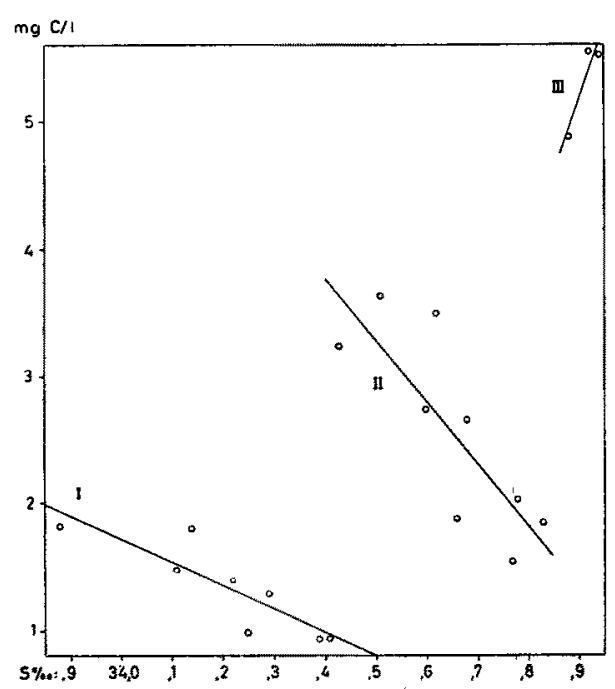

Abb. 7. C/S-Diagramm der Stationen 934 bis 955 von FFS „Anton Dohrn“ (Mittel der gesamten Wassersäule).

Um wie große Mengen an organischer Substanz es sich handelt, zeigt der Vergleich mit der auf Grund von $\mathrm{O}_{2}$-Änderungen ermittelten Produktion im Englischen Kanal (AткıNs, 1923). Rechnen wir einmal überschlägig im Untersuchungsgebiet mit $3 \mathrm{mg} \mathrm{C/1} \mathrm{-} \mathrm{ähnliche} \mathrm{Werte} \mathrm{gibt} \mathrm{z}$. B. auch KAY (1954 b) aus der westlichen Ostsee an - oder bei $40 \mathrm{~m}$ Wassertiefe $120 \mathrm{~g} \mathrm{C} / \mathrm{m}^{2}$. Die Jahresproduktion im Kanal beträgt etwa $60 \mathrm{~g} \mathrm{C} / \mathrm{m}^{2}$, so daß selbst unter der Annahme, die gesamte erzeugte organische Substanz würde in Lösung gehen, mit einer mittleren Lebensdauer von zwei Jahren gerechnet werden muß, in Wahrheit also sicherlich noch mit erheblich mehr. (Der schnellere Abbau bei Zehrungsversuchen ist durch die veränderten Umweltbedingungen - vor allem die große Oberfläche der Gefäße - bedingt.) SteEmann Nielsen (1955) gibt für die gelösten organischen Substanzen der freien See ebenfalls eine sehr langsame Zersetzung an. Der gelöste organische Kohlenstoff ist mithin eine 
relativ konservative Größe hinsichtlich der in einem Wasserkörper stattgefundenen Planktonproduktion.

Wir haben es demnach mit drei Wasserarten zu tun, die aus unterschiedlichen Produktionsgebieten stammen. Es wäre durchaus denkbar, daß I aus dem Kanal kommt, II vom Norden her an der Ostküste Englands eingeströmt ist und III altes Nordseewasser darstellt.

Ein anderes Bild hinsichtlich der Zersetzungsgeschwindigkeit geben hingegen die zumeist gelösten organischen Phosphorverbindungen (ARMstrong und Harvey, 1950). Während sich im Englischen Kanal im Mai im Mittel $7,8 \mathrm{mg}$ organischer $\mathrm{P} / \mathrm{m}^{3}$ im Wasser finden, um dann während des Sommers etwa $6 \mathrm{mg} \mathrm{P} / \mathrm{m}^{3}$ zu betragen, fällt dieser Wert bis zum Januar auf 2,3 $\mathrm{mg} \mathrm{P} / \mathrm{m}^{3}$ ab. Der Abbau der organischen Phosphorverbindungen erfolgt also wenigstens hinsichtlich der Phosphorkomponente wesentlich schneller als der der gesamten organischen Substanz. Eine Beteiligung von im Seewasser gelösten Fermenten an dieser raschen Phosphatabspaltung ist nach HofrmanN (1956) nicht anzunehmen.

\section{$\mathrm{Zusammenf}$ assung}

Es wird ein Verfahren zum oxydativen Nachweis von organischer Substanz im Seewasser beschrieben und seine praktische Bedeutung an einer Untersuchung in der Nordsee erläutert.

\section{Literaturverzeichnis}

Armstrong, F., und Harvey, H., 1950: The cycle of phosphorus in the waters of the English Channel. Journ. Mar. Biol. Assoc. 29.

$\Lambda$ tkins, W. R. G., 1923: The hydrogen ion concentration of sea water in its relation to photosynthetic changes. Part II. Journ. Mar. Biol. Assoc. 13.

Dietrich, G., 1954: Verteilung, Ausbreitung und Vermischung der Wasserkörper in der südwestlichen Nordsee auf Grund der Ergebnisse der "Gauss"-Fahrt im Februar/März 1952. Ber. Dtsch. Wiss. Komm. Meeresf. 13, 2.

Gillbricht, M., 1952: Untersuchungen zur Produktionsbiologie des Planktons in der Kieler Budht I. Kieler Meeresf. 8, 2.

Hahn, F. L., und Wind isch, H., 1922: Das Altern maßanalytischer Thiosulfatlösungen. Ber. Dtsch. Chem. Ges. 55.

Hof $\mathrm{f} \operatorname{mann}, \mathrm{C} ., 1956$ : Untersuchungen über die Remineralisation des Phosphors im Plankton. Kieler Meeresf. 12, 1.

Kalle, K., 1939: Einige Verbesserungen zur Bestimmung des gelösten Sauerstoffs im Meerwasser. Ann. Hydrogr. u. Marit. Meteorol. 67.

$\mathrm{K}$ a y, H., 1954 a: Eine Mikromethode zur chemischen Bestimmung des organisch gebundenen Kohlenstoffs in Meerwasser. Kieler Meeresf. 10, 1.

- $1954 \mathrm{~b}$ : Untersuchungen zur Menge und Verteilung der organischen Substanz im Meer. wasser. Kieler Meeresf. 10, 2.

Krey, J., 1939: Die Bestimmung des Chlorophylls in Meerwasserschöpfproben. Journ. du Conseil 14, 2.

- 1951: Quantitative Bestimmung von Eiweiß im Plankton mittels der Biuretreaktion. Kieler Meeresf. 8, 1.

Ohle, W., 1953: Die chemische und elektrochemische Bestimmung des molekular gelösten Sauerstoffs der Binnengewässer. Intern. Vereinig, f. theor. u. angtw. Limnologie, Mitt. 3.

Ruppin, E., 1904: Ober die Oxydierbarkeit des Meerwassers dutch Kaliumpermanganat. Cons. Perm. Intern. Explor. Mer. Public, de Circonstance 20. 
Stecmann Nielsen, E., 1955: The production of antibiotics by plankton algae and its effect upon bacterial activities in the sea. Deep Sea Res. Suppl. to 3 (Bigelow).

Sverdrup, H., Johns on, M., und Fleming, R., 1946: The Oceans. New York.

Winkler, L. W., 1931: Ausgewählte Untersuchungsverfahren für das chemische Laboratorium. Die Chemische Analyse (W. Böttger). Verl. Ferd. Enke, Stuttgart.

Nachtrag zum Aufsatz Gillbricht

\section{Oxydativer Nachweis von organischer Substanz}

Nach Drucklegung der Arbeit wurden mit Unterstützung der Deutschen Wissenschaftlichen Kommission für Meeresforschung auf einer Fahrt von FFS "Anton Dohrn" in die Gewässer zwischen Island und Grönland (April 1957) Untersuchungen über die Menge der oxydierbaren organischen Substanz durchgeführt. Die gefundenen Werte waren weit niedriger als erwartet (im Durchschnitt $<0,5 \mathrm{mg}$ C- Äquivalent/1), so daß eine Erhöhung der Empfindlichkeit der Methode wenigstens bei geringen Konzentrationen wünschenswert schien.

Es ließ sich nun zeigen, daß bei gleicher $\mathrm{NaOH}-M e n g e(0,8 \mathrm{~g})$ die Seewassermenge unbedenklich auf $40 \mathrm{ml}$ gesteigert werden kann, ohne daß die Oxydationswirkung des Permanganats hierdurch vermindert wird. Unter dieser Bedingung tritt dann beim späteren Ansäuern mit $3 \mathrm{ml} \mathrm{H}_{2} \mathrm{SO}_{4}$ (1:3) keine bedenkliche Neutralisationswärme auf. Die absolute Genauigkeit der Methode bleibt so erhalten, die mittlere Streuung der Messungen beträgt mithin etwa $\pm 0,03 \mathrm{mg} \mathrm{C}$-Äquivalent/l.

Nach Möglichkeit sollte das Seewasser sofort nach der Entnahme mit $\mathrm{NaOH}$ versetzt und dann nicht zu lange gelagert werden. Wird erst angesäuert, so erhöht sich mit der Menge der zugegebenen Chemikalien die Gefahr einer Verunreinigung. Notfalls können jedoch zunächst $1 \mathrm{ml}$ verdünnte $\mathrm{H}_{2} \mathrm{SO}_{4}$ ( $150 \mathrm{ml}$ aqua dest. $+90 \mathrm{ml} \mathrm{H}_{2} \mathrm{SO}_{4}$ ) und später $1,4 \mathrm{~g} \mathrm{NaOH}$ zugesetzt werden.

Bis $2,5 \mathrm{mg} \mathrm{C}$-Äquivalent/l lassen sich so durch Zugabe von $5 \mathrm{ml}$ einer Permanganatlösung von $1 / 50 \mathrm{val} / 1$ nachweisen. Eine stärkere Verdünnung des Permanganats ist nicht ratsam, jedoch lassen sich noch bei einiger Vorsicht mit 1/20 val/1 Permanganat bis zu $6 \mathrm{mg} \mathrm{C}$-Äquivalent/l bestimmen. In diesem Falle führt das Umfüllen der recht starken Lösung zu unkontrollierbaren Jodverlusten und damit zu großen Streuungen. Um dies zu vermeiden, wird die Probe nach dem Kochen vor dem Ansäuern zunächst quantitativ (zuletzt durch saures Nachwaschen) in eine $100 \mathrm{ml}$ Glasstopfenflasche gespült und später darin titriert. So läßt sich die Genauigkeit der verdünnteren Permanganatlösung erreichen.

Die Auswertung geschieht dann wie folgt:

$$
\begin{array}{ll}
\mathrm{mg} \mathrm{C}-\not \text { Aquivalent } / 1 & =18,77 \cdot \frac{(\mathrm{K}-\mathrm{P})}{\mathrm{T}} \\
\mathrm{mg} \mathrm{KMnO}_{4} / \mathrm{l} & =197,5 \cdot \frac{(\mathrm{K}-\mathrm{P})}{\mathrm{T}}
\end{array}
$$

